\title{
Innovative policies for implementing intelligent speed adaptation on urban roads
}

\author{
V. Mehta, W. Walker, V. Marchau \& B. Agusdinata \\ Faculty of Technology, Policy and Management, \\ Delft University of Technology, the Netherlands
}

\begin{abstract}
Safety is of utmost importance in urban road traffic. Many studies reveal that speed is the major factor contributing to road accidents. In the Netherlands, $50 \%$ of the speed offences and $65 \%$ of the accidents occur on urban roads. Research by the Dutch Ministry of Transport indicates that the elimination of speeding would reduce accidents and fatalities by $21 \%$ and hospitalized victims by $15 \%$, and provide benefits to the Dutch economy of 300 million euros per year. Implementation of Intelligent Speed Adaptation (ISA) seems to be a promising way to meet these targets. Although ISA is a tested and proven technology, its implementation is impeded by many uncertainties, including its acceptance by drivers, its effects on driving behavior, and its effectiveness in real world driving conditions (as opposed to 'laboratory' conditions). As a result, policymakers need a way to implement ISA so that its safety benefits can be obtained despite the existence of uncertainties that cannot be reduced in advance. This paper proposes an innovative, adaptive approach for implementing ISA that would permit implementation to proceed while knowledge was gained about the uncertainties over time (i.e., the level of uncertainty was reduced). Under the adaptive approach, actions to deal with the certain vulnerabilities of the policy are implemented at the same time as the policy, and uncertain vulnerabilities are monitored and actions taken as required. This approach allows adaptations to the policy over time as knowledge about ISA is gained and critical events for ISA implementation take place. In particular we propose to begin by implementing ISA (Dynamic Voluntary) for young car drivers between 18-34 years of age and on unsafe roads i.e. urban roads ( 30 to $80 \mathrm{~km} / \mathrm{h}$ ) and to prepare to adapt the policy over time.

Keywords: intelligent speed adaptation, uncertainties, adaptive policymaking.
\end{abstract}




\section{Introduction}

Safety is one of the most serious problems facing road transport. Many technological solutions to deal with safety problems have been proposed, but few have been implemented because of uncertainties associated with the technologies, the behaviour of drivers who would be using the technologies, and how people might value the costs and benefits. This paper focuses on one such technology - Intelligent Speed Adaptation (ISA) and proposes an innovative approach for dealing with the uncertainties surrounding its implementation.

\subsection{The speed factor in road accidents}

Speed is one of the primary causes of traffic accidents and traffic fatalities. Faster driving speeds result in larger collision speeds and thus more serious outcomes from accidents. Faster driving speeds also provide less time to process information and to act on it, and also the braking distance is longer, reducing the possibility of avoiding a collision on road. Hence the chances of accidents increase on the road due to higher speed. Speeding of vehicles leads to the loss of life and property and also large costs for emergency services, repair bills and increased insurance premiums. Many studies show that the majority of road accidents are due to inappropriate vehicle speeds. It appears from the figures that speed contributes to one third of the fatal and serious crashes. Two in three drivers exceed the speed limit in urban areas, and one in two drivers exceed the limit on single lane rural roads in the EU. Better speed management, which can reduce average speeds by $5 \mathrm{~km} /$ hour, could save as many as 9000 lives annually in the EU [1]. At $80 \mathrm{~km} / \mathrm{h}$, the chance that a car occupant is killed in an accident is about 20 times greater than in an accident at a speed of $30 \mathrm{~km} / \mathrm{h}$. There is an exponential relationship between speed and accident rate [2].

\subsection{Safety on urban roads}

The above relationship suggests that the motorways should have a higher accident rate than urban roads because of the higher speeds. But this is not the case. It can be seen in Figure 1 that motorways have the lowest crash rate and, as the speed increases, the crash rate increases less rapidly as compared to rural and urban roads. The reverse is also true: the same drop in speed has a larger safety effect on rural and urban roads than on motorways [3]. This is because of the more complex and dynamic traffic environment on rural and urban roads, such as encounters with different types of road users - pedestrians, cyclists, mopeds, etc. Drivers on urban and rural roads have to deal with a variety of driving situations at different four-corner junctions and $\mathrm{T}$ junctions, where the traffic comes from different directions and with less predictable behaviour. Because of the dynamic traffic environment, a driver has to process more information, distracting him while driving. Speeding in such a condition would increase the accident rate. Compared to urban and rural roads, the traffic on motorways is more homogenous. There are no pedestrian and cyclists activities on the motorways. The traffic flow is in single direction, and there are also no crossroads, making 
the traffic behaviour more predictable. Also the motorways have higher design standards compared to urban and rural roads. Hence the rate of increase in accidents as a function of speed is lower on motorways than on urban and rural roads.

In the Netherlands in 2003, there were 1088 traffic fatalities and 18,000 or more serious injuries on roads [4]. Also, the overall number of speed offences in the Netherlands has been increasing since 1996, especially on urban throughroads. More than $70 \%$ of the speeding offences are in urban areas on $50 \mathrm{~km} / \mathrm{h}$ double carriageways. Also, $65 \%$ of all accidents take place on urban roads [5].

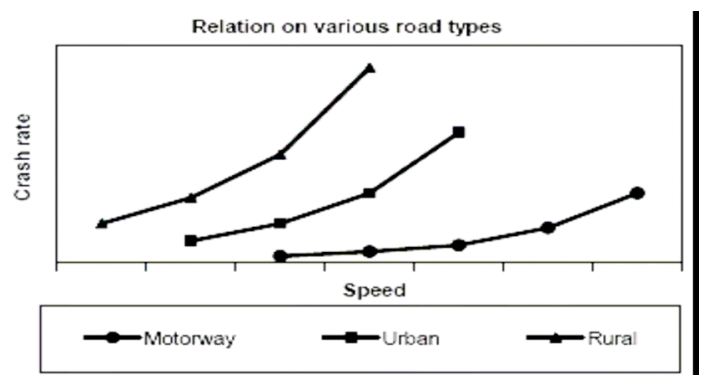

Figure 1: Relation between speed and crash rate on different road categories (Source: [3]).

\subsection{Measures for improving road safety}

A study into the costs and benefits of lowering car speeds in the Netherlands has shown that enforcing the current speed limits would lead to a reduction in the number of hospitalised victims by $15 \%$ and in the number of fatalities by $21 \%$, which would produce economic benefits of 300 million euros per year [6]. In order to achieve the targets of reducing accidents due to speed, many policy actions have been taken, such as road categorisation, designed speed limits, introduction of black boxes, road design, optical lane narrowing, posting fixed and dynamic local speed limits on road, etc. Implementing these actions did not help achieve the targets of accident reduction due to speed. The use of Intelligent Speed Adaptation - one type of Advanced Driver Assistance System - seems to be a good way to achieve the targets. ISA informs the driver and/or controls the speed of the vehicle in accordance with the official posted speed limit. Use of Intelligent Speed Adaptation (ISA) would reduce the average speed and speed distribution of vehicles on the road. Tests results based on simulation studies and small pilot experiments in UK Leeds show that ISA with $100 \%$ penetration level would decrease the number of fatal accidents by $24 \%, 32 \%$ and $59 \%$ for Advisory, Voluntary and Mandatory ISA respectively. It also has other positive effects on the transport system in terms of emission levels, fuel consumption and traffic flow. Also, tests in the Netherlands confirm that implementing ISA as a policy option would help to attain the road safety goals set by the Ministry of Transport, Public Works and Water Management. 
ISA has its advantages, but on the other side there are uncertainties attached to the idea of implementing ISA. Because it is a new technology, not much is known about implementing it on a large scale in a real world environment. Test results have been based on small scale implementation or simulation studies, assuming optimal functioning of the system as per the design and $100 \%$ penetration level, implying limited real world validity. Because of this, the real world large scale implementation of ISA is surrounded by many types of uncertainties. This includes its acceptance by the user, its effects on driving behaviour, its effectiveness in real world driving conditions, and its reliability. Hence there are large uncertainties attached to implementation of ISA, and it becomes important to deal with these uncertainties for smooth implementation of ISA. This paper proposes an innovative policymaking approach to develop policies that would cope with the uncertainties surrounding the implementation of ISA. In Section 2 the need for innovative policymaking and an adaptive policymaking approach is discussed. In Section 3 an adaptive policy is developed.

\section{Need for innovative policymaking}

Policymaking concerns making choices or developing strategies regarding a system in order to change the system outcomes in a desired way, in accordance with a set objective. Public policymaking not only develops strategies considering the interactions of the physical elements of the system, but also must take into account the behavioural mechanisms underlying these interactions [7]. Therefore it is important to deal with uncertainties, since they might have negative social consequences that would affect the success of the policy's implementation.

\subsection{Shortcomings of traditional policymaking}

In traditional policymaking, uncertainties are often handled using scenarios, and the selection of a policy is based on its performance across different scenarios. The problems with this traditional approach are:

- The policy is based on assumptions about the future. If the assumptions were to fail or if critical uncertainties were overlooked in developing the scenarios, the policy might fail.

- $\quad$ The policy developed would be best for specific scenarios that are fairly certain not to occur, since any given scenario has a probability zero of actually occurring. [8].

- The policy developed is static and inflexible. Such a fixed policy can fail because it doesn't adapt as the world changes.

Considering the uncertainties surrounding the implementation of ISA and the shortcomings of traditional policymaking for handling uncertainties, it can be concluded that the traditional policymaking approach in handling uncertainties related to ISA implementation is vulnerable to policy failure. This is because ISA is a new technology and not much is known about its behaviour and 
performance on large scale. Also when ISA is implemented, new knowledge and information would be gained and the traditional approach doesn't incorporate ways of monitoring, learning, and applying the new knowledge that can be used to refine the policies that are implemented. Also, the policy developed using the traditional approach would be static. To be successful, the policy should be able to adapt to changing circumstances. To overcome these problems of traditional policymaking, an innovative adaptive policymaking approach is developed for implementing ISA.

\subsection{Adaptive policymaking approach}

In adaptive policymaking, the policy is updated over time with respect to the changes in the system and as new information becomes available. A policy developed using the adaptive approach is designed to be incremental, adaptive, and conditional [8]. Part of the policy is implemented now and the rest in the future, as and when required, based on the new information and knowledge. This approach has a systematic way of monitoring the environment and adjusting the pieces of the policy to the new circumstances as new information about events/developments becomes available. The policies developed by such an approach are more flexible in order to handle uncertainties. The approach is based on identifying in advance the conditions of success and unknown events or vulnerabilities that could make the policy fail, and specifying the actions to be taken in response to them. These vulnerabilities are also monitored using signposts. Trigger levels for the signposts are established and new policy actions are taken if and when the trigger levels are reached. Hence, policy actions are taken when required and not on an ad hoc basis. (The adaptive approach has been developed by Warren Walker, Jonathan Cave and Adnan Rahman of RAND [8].) The framework for the adaptive policymaking procedure is shown in Figure 2. Using this framework, an adaptive policy is developed for implementing ISA in the following section.

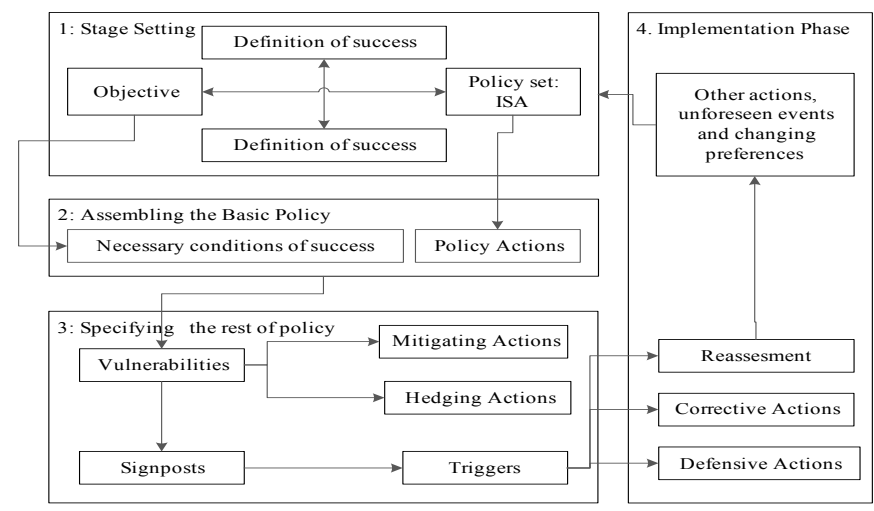

Figure 2: Adaptive policymaking procedure. 


\section{Developing an adaptive policy for ISA}

Step 1 in the adaptive policymaking approach includes the identification of objective, constraints, and available policy options. The objective for ISA was identified by the Dutch Ministry of Transport, Public Works and Water Management. The objective is a reduction by $25 \%$ in fatalities and $22 \%$ in serious injuries due to road accidents by 2010 (as compared to the figures of 2003). Considering the speed-accident relationship and the speed offences in the Netherlands, Intelligent Speed Adaptation has been identified as a policy option. Test results in the Netherlands on ISA predict a $34 \%$ reduction in fatal accidents and a $27 \%$ reduction in injuries as a result of large scale implementation of Mandatory ISA on $30 / 50 \mathrm{~km} / \mathrm{h}$ roads [1]. Hence, ISA is set as a policy option that would help achieve the objective set. The constraints are the limits to the policy. The major constraint would be financial. The other constraints are that congestion, travel, and local pollution should not increase because of the implementation of ISA.

In Step 2, the basic ISA policy is specified and the necessary conditions for its success are identified. To start with, we propose to implement a suitable type of ISA for unsafe roads, unsafe vehicles, unsafe traffic conditions, and unsafe drivers. As compared to rural roads $(80-100 \mathrm{~km} / \mathrm{h})$ and motorways (100$120 \mathrm{~km} / \mathrm{h})$, urban roads $(30-80 \mathrm{~km} / \mathrm{h})$ are more dangerous. $64.5 \%$ of road traffic accidents are on $50 \mathrm{~km} / \mathrm{h}$ roads [4]. Also, there are mixed traffic situations on urban roads as compared to other categories, making them more vulnerable and unsafe. The test results also show that ISA has larger effect on 30,50 and 70 $\mathrm{km} / \mathrm{h}$ roads as compared to 80,100 and $120 \mathrm{~km} / \mathrm{h}$ roads [9]. It is also visible from the statistics that the number of persons killed in traffic participation is highest for cars as compared to other vehicles and for drivers between the ages of 18-24 years followed by 25-34 years. Hence, young car drivers are considered to be vulnerable. This policy proposes the use of voluntary ISA because even though mandatory ISA has larger positive effects, it would be less acceptable by the users that their speed is controlled by the vehicle and not by them. User acceptance is very important for the success of ISA. Also a constrained top speed might produce complications on the road while overtaking or in dangerous situations, in which the driver needs to accelerate. Also, because of mixed traffic conditions on urban roads, incidents on urban roads, and change in weather condition, we propose to implement dynamic ISA. The communication of the speed limits to the vehicle will be by GPS with built in speed limits on CD ROM; at places where there is lack of GPS signal (e.g. in tunnels or areas surrounded by tall buildings), beacons will be installed. Hence, the basic policy specified for implementation is dynamic voluntary ISA on urban roads for young car drivers on a voluntary basis.

The necessary conditions are the ones that will be monitored in the future to ensure the success of the basic policy. One of the necessary conditions for success for the basic policy would be that factors like speed, speed distribution, and speed offences on urban roads should decrease because of ISA implementation. Also the other condition of success is that there should be 
operational reliability of ISA technology, i.e. reliable communication systems and accurate speed data. The most important condition for success is user acceptance. There should be at least a $60 \%$ penetration level of ISA technology.

In Step 3 the remaining part of the policy is specified. It is this part that makes the policy adaptive. This step includes two types of analysis: (1) the identification of vulnerabilities, together with the specification of actions to be implemented when the basic policy is implemented, and (2) the translation of the necessary conditions of success and vulnerabilities into signposts. These signposts will be monitored in the future as implementation proceeds to ensure the smooth implementation of ISA. Any undesired change in the signpost level would trigger a change in policy.

One of the uncertain vulnerabilities is a low penetration level or user acceptance of ISA, since the policy is being implemented for young drivers. The test results show that ISA would have maximum positive effects in terms of accident reduction if the penetration level is $60 \%$ to $100 \%$ [10]. The user acceptance depends on factors like costs, reliable operation of the ISA system, comfort level while driving, etc. If there is low user acceptance, there would be a low level of accident reduction. To attain the required penetration level, the hedging action to start with could be conducting advertising, workshops, and campaigns about ISA in the media, universities, and newspapers with an idea/concept of safety. This would help the citizens and the users to know the benefits of the technology. Other actions could be to provide incentives to the initial users of the technology, such as by providing equipment at a subsidised rate, lowering insurance premium, providing free parking, lowering taxes, etc. It should be made clear what incentives should be provided, considering the financial constraints and level of user acceptance and under what circumstances (i.e. now and also in future). Providing incentives would also be a help to young drivers. Along with these two actions, information related to malfunctions on the road should also be monitored to keep check on the reliability of the technology.

Another uncertain vulnerability is that, due to constant information supply while driving on the road, the driver might become frustrated or distracted. This could lead to risky situations on urban roads. Also, drivers might lose vigilance and decrease the distance to other vehicles because of increased reliability on the system, which could be hazardous and result in accidents. To curb such a dangerous situation, a hedging action could be arranging training programs for ISA users and drivers using real time simulation for different driving conditions with voluntary dynamic ISA. This would make the driver accustomed to ISA and its effects before driving on the road. This would also help the driver to understand the interface with the ISA system while driving, and the driver can also customize its interface. This could be a part of driving school exercise. Also, user reports can be developed that would help the ISA implementers to understand the problems faced by ISA users while driving on urban roads. This can be done by installing black boxes in the vehicles that would store information such as vehicle speed, overriding situations, malfunctions, driver behaviour, etc. It could also be done by surveying ISA users. The information 
gained from the users can then be used to develop strategies to improve user acceptance and to refine ISA operational reliability.

The penetration level of ISA is a signpost to check the user acceptance. It would help us to understand whether actions need to be taken to attain the desired penetration level of ISA. Another signpost for the user acceptance could be a user report that could help us to understand the ergonomics problems the user faced while driving, which could be used to refine the ISA system operation.

Another important signpost would be the number of accidents involving ISA users. Accidents can be classified into fatalities and injuries. This information would help us to take necessary actions if required.

To decide upon a critical value for a signpost (i.e. the trigger level), a target value has to be calculated considering the success criteria and time to attain the desired value. This target value can be changed when required considering the success attained in past and what is to be attained in future. For example, for the signpost penetration level, the desired value might start with an increase of $1 \%$ per month with a time limit of 5 years. If at the end of one year an $8 \%$ penetration level is attained instead of $12 \%$, a new desired increase in penetration of $1.3 \%$ per month might be established for the next 4 years. Trigger levels for policy actions should be based on target values and regret levels (see Figure 3). During implementation, if for a signpost the regret level 1 is reached, defensive actions are taken; corrective actions are taken for more critical levels. Also the conditions under which a reassessment of the policy would be made should be identified before beginning to implement the policy. For example, if the number of accidents on the road doesn't change or if they have increased within a stipulated time and the expected outcomes are not being attained or are even getting worse, then the policy has to be reassessed. Figure 3 shows the hypothetical example for the signposts and triggers related to the rate of penetration. A similar figure can be drawn for the other signposts (e.g. change in speed offences and accidents involving ISA users).

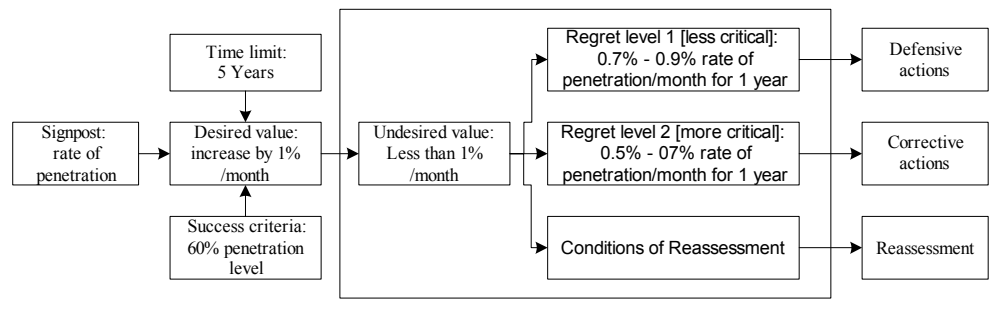

Figure 3: $\quad$ Identifying and handling triggers.

Step 4 of the adaptive approach is the implementation phase. During the implementation phase the information is collected for each signpost. The information gathered is compared to the critical level for each signpost. Defensive and corrective policy actions are taken as per the regret level i.e. level 1 or 2 . For example, defensive actions to increase the rate of penetration could be 
investments in R\&D to solve the technical operation problems of ISA. Other defensive actions could be an increase in speeding fines and installation of speed cameras. If the regret level is more critical, then some corrective actions can be taken, such as implementing ISA on a mandatory basis and increasing the scope of the policy by involving drivers of all ages and for all road categories. Also the user report developed could help to develop defensive actions. For example, if a frequent problem of hardware breakdown is reported, the system can be repaired free or replaced. Also, problems leading to breakdowns can be identified and solved.

For the signpost "accidents involving ISA users" a defensive action could be the deployment of accident response teams in certain identified dangerous locations on urban roads. In case of an increase in accidents due to riskier gap acceptance, a defensive action could be installation of rear and head on crash warning control systems in the vehicles; a corrective action could be a shift of the basic policy from dynamic voluntary ISA to static or variable advisory ISA.

If neither defensive nor corrective actions are sufficient to achieve the specified success conditions, the policy would have to be reassessed.

\section{Conclusions}

The adaptive policymaking approach is a more logical and systematic procedure to deal with uncertainties compared to traditional policymaking for ISA implementation. Considering the large potential positive impact of ISA on safety and the large uncertainties involved in large scale real world implementation, adaptive policymaking seems to be a promising approach.

\section{References}

[1] J. H. Kraay, The Netherlands traffic and transport plan: Road Safety with a special focus on speed behaviour. Proceedings of the $15^{\text {th }}$ ICTCT workshop, Nagoya, Transport Research Centre (AVV), Ministry of Transport, the Netherlands, 2002.

[2] G. Nilsson, The effects of speed limits on traffic crashes in Sweden. Proceedings of international symposium on the effects of speed limits on traffic crashes and fuel consumption, Dublin. Organisation for Economy, Co-operation, and Development (OECD), Paris, 1982.

[3] SWOV fact sheets, http://www.swov.nl/rapport/factsheets/fs speed.pdf

[4] Road Safety in the Netherlands: Key Figures Edition 2004 - Ministry of Transport, Public Works and Water management, www.rws-avv.nl.

[5] V. Mehta, Dealing with Uncertainties in Implementing Intelligent Speed Adaptation - An Adaptive Policymaking Approach. Masters Thesis, Faculty of Technology, Policy and Management, Delft University of Technology, Dec. 2005.

[6] Alex van Loon and Lies Duynstee, Intelligent Speed Adaptation: A successful test in the Netherlands. Transport Research Centre (AVV), 
Ministry of Transport, the Netherlands, Feb 2001. http://www.rwsavv.nl/pls/portal30/docs/911.PDF

[7] V.A.W.J. Marchau and W.E. Walker, Dealing with Uncertainty in Implementing Advanced Driver Assistance Systems: An Adaptive Approach. Integrated Assessment 4(3) 2003.

[8] W.E. Walker, S.A. Rahman and J. Cave, Adaptive Policies, Policy Analysis, and Policymaking. European Journal Oper, Res. 128(2) 2001.

[9] I. Wilmink, E. Versteegt, R. Liu and G. Hegeman, Network Effects and Policy Impacts of ISA - A Micro Simulation Analysis. ITS Congress Conference, Nagoya, 2004.

[10] O. Carsten, S. Jamson, K. Chorlton and M. Fowkes, Intelligent Speed Adaptation - Literature Review and Scoping Study. University of Leeds, MIRA Ltd. and Tfl, January 2006. 